\title{
Cordycepin inhibits cell growth and induces apoptosis in human cholangiocarcinoma
}

\author{
C. WANG ${ }^{1}$, Z. P. $M A O^{1}$, L. WANG ${ }^{1}$, F. H. ZHANG ${ }^{1}$, G. H. WU' , D. Y. WANG ${ }^{1}$, J. L. SHI ${ }^{2, *}$ \\ ${ }^{1}$ Department of Oncology Surgery, the Affiliated Hospital of Logistics University of PAP, Tianjin 300162, China; ${ }^{2}$ Department of thoracic surgery, \\ the Affiliated Hospital of Changzhi Medical College, Changzhi Shanxi 046000, China
}

${ }^{*}$ Correspondence: dr_jialunshi@163.com

Received January 12, 2017 / Accepted March 8, 2017

\begin{abstract}
The purpose of this study was to explore the role of cordycepin in human cholangiocarcinoma (CCA) cell growth and apoptosis. In the present study, colony formation assay, cell-counting kit-8 (CCK-8) assay and tumor xenograft experiment were performed to evaluate the effect of cordycepin on human CCA cell growth in vitro and in vivo; flow cytometric analysis was performed to evaluate the effect of cordycepin on cell apoptosis; quantitative real-time reverse transcription PCR (qRTPCR) and western blot assays were performed to evaluate the expression levels of Caspase-3, Bcl-2 and Bax. The results showed that cordycepin inhibited cell growth in QBC939 and RBE cells in vitro and it could also inhibit QBC939 cells growth in vivo. Furthermore, the flow cytometric analysis, qRT-PCR and western blot assays showed that cordycepin could trigger QBC939 and RBE cells apoptosis by regulating the expression levels of Caspase-3, Bcl-2 and Bax. And we proposed that cordycepin could inhibit human CCA cell growth in vitro and in vivo, while, this function is related to the induction of cell apoptosis.
\end{abstract}

Key words: Cordycepin, cholangiocarcinoma, cell proliferation, apoptosis

Cholangiocarcinoma (CCA) is a biliary tract malignancy arising from the epithelium of biliary tree. After hepatocellular carcinoma, CCA is the second most common primary liver malignancy [1]. Due to the lack of specific symptoms and signs, CCA patients are usually diagnosed at its advanced stage and lose the opportunity to undergo the radical surgery, which is the most effective treatment for CCA. With the addition of the resistance to chemotherapy, the 5-year survival rate of CCA is extremely low [2]. The epidemiological studies indicated that the morbidity and mortality of CCA was rising over the past decade, especially in China $[3,4]$. Therefore, the development of new treatments or drugs to improve the outcome of CCA is of paramount necessity.

Cordycepsmilitaris, a fungus that parasitizes Lepidoptera larvae, has been widely used as one kind of folk tonic food or crude drug in the tradition [5]. Cordycepin, also termed 3 '-deoxyadenosine, is a chief ingredient and the active component isolated from Cordycepsmilitaris, has been reported to have various anti-tumor activities, including cell proliferation inhibition, cell migration inhibition and apoptosis induction [6-8]. For its low toxicity to normal cells, cordycepin has been regarded as a preventative ortherapeutic agent to some tumor cells $[9,10]$. Some studies have reported its anti-tumor activities in human cancer of lung, liver, colorectum and etc $[7,11$, 12]. However, as far as we know, the role of cordycepin in the development of CCA remains unknown.

In this study, we explored the effect of cordycepin on the human CCA cell lines in vitro and in vivo, and put forward that the inhibiting effect of cordycepin on human CCA cell growth is, at least in part, through inducting human CCA cell apoptosis.

\section{Materials and methods}

Cell culture. The human CCA cell lines QBC939 and RBE were separately cultured in DMEM high-glucose medium (HyClone, USA) and RPMI-1640 medium (Gibco, USA) supplemented with 10\% feral bovine serum (Gibco, USA). The cells were cultured under an atmosphere of $5 \% \mathrm{CO}_{2}$ in a humidified incubator at $37^{\circ} \mathrm{C}$.Cordycepin was bought from Sigma-Aldrich (Saint Louis, USA), and we treated QBC939 and RBE cells with cordycepin at the concentration of $25 \mathrm{mM}$.

Quantitative real-time reverse transcription PCR (qRTPCR). Total RNA was extracted from human CCA cell using 
TRIzol reagent (Invitrogen, USA). RNA quantity and quality were identified by NanoDrop 2000c (Thermo Scientific, USA). For each sample, $1 \mu \mathrm{g}$ of total RNA was reverse transcribed to cDNA using a reverse transcription kit (Promega, USA). The PCR reaction was conducted to evaluate the mRNA expression levels by using the SYBR Green reagent (Applied Biosystems, USA) and ABI7500 PCR machine (Applied Biosystems, USA). The relative mRNA expression levels were calculated using the $2^{-\Delta \Delta \mathrm{Ct}}$ method, ACTIN was used as an internal control for normalization. Each experiment was conducted in triplicate. The primer sequences were listed as follows: ACTIN-F, 5'-AAAGACCTGTACGCCAACAC-3', ACTIN-R, 5'-GTCATACTCCTGCTTGCTGAT-3'; Caspase3-F, 5'-GAAATTGTGGAATTGATGCGTGA-3', Caspase3-R, 5'-CTACAACGATCCCCTCTGAAAAA-3';
Bcl2-F，5'-GGTGGGGTCATGTGTGTGG-3', Bcl2 R, 5'-CGGTTCAGGTACTCAGTCATCC-3'; Bax-F, 5'-CCCGAGAGGTCTTTTTCCGAG-3', Bax-R, 5'-CCAGCCCATGATGGTTCTGAT-3'.

Cell proliferation assay. Cell proliferation assays were conducted by using Cell Counting Kit-8 (Dojindo, Japan). Human CCA cell was plated in 96-well plates in triplicate at approximately 1000 cells per well and cultured in the medium. Then, the cells were treated with cordycepin. After 48 hours, the $450 \mathrm{~nm}$ absorbance was measured to assess the cell viability using a microplate reader. Each experiment was conducted in triplicate.

Colony formation assay. The human CCA cell was plated in 6-well plate atapproximately 1000 cells per well and treated with cordycepin. After culture for 2 weeks, the cells were fixed
A

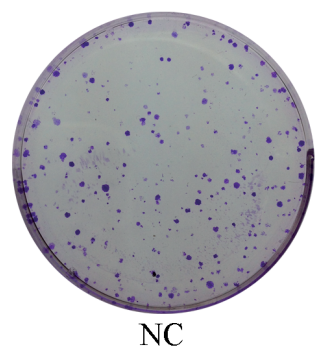

$\mathrm{C}$

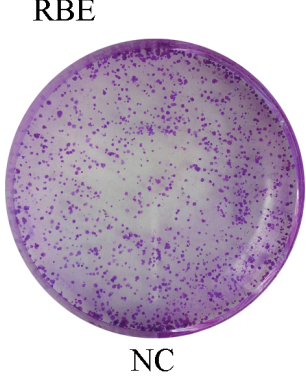

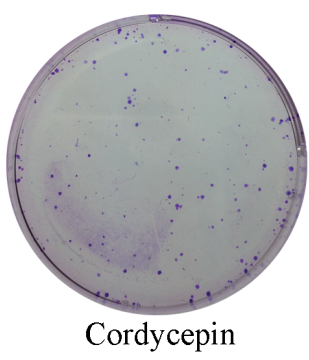

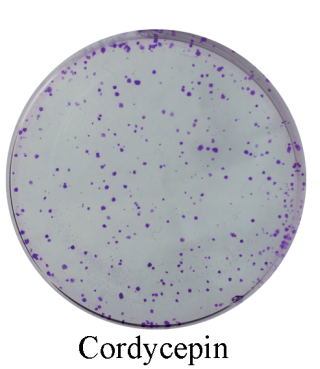

B

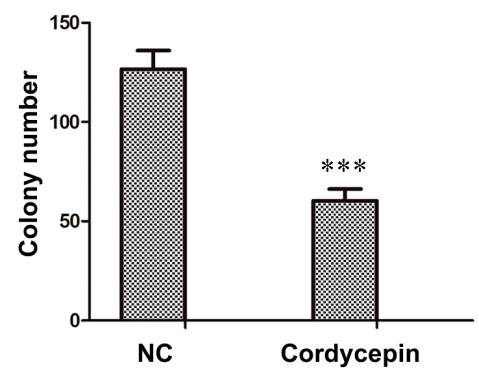

$\mathrm{D}$

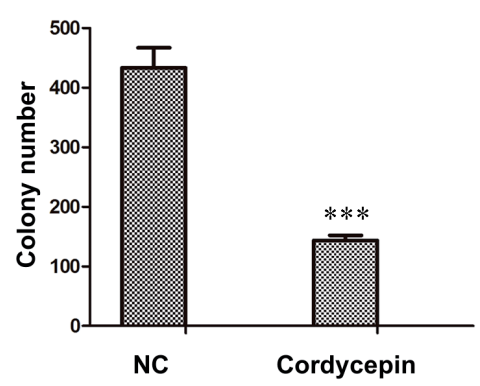

$\mathrm{E}$

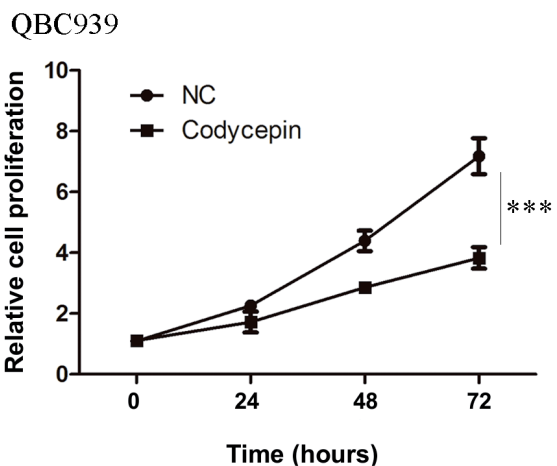

$\mathrm{F}$

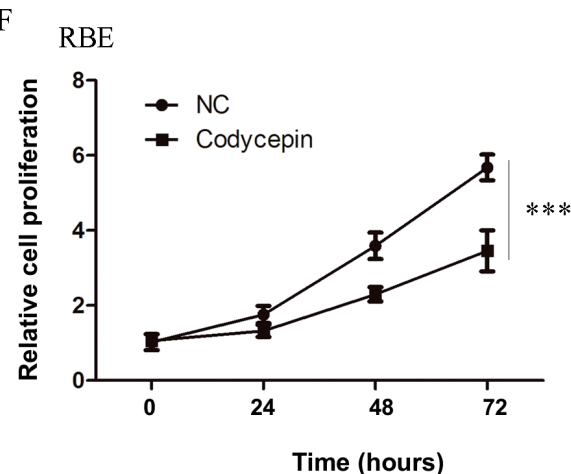

Figure 1. Cordycepin inhibits growth in human CCA cell in vitro. (A and B) Cell colony formation assay in human CCA cell line QBC939 treated with cordycepin was shown. (C and D) Cell colony formation assay in cell line RBE treated with cordycepin was shown. (E) CCK-8 assay in cell line QBC939treated with cordycepin was shown. (F) CCK-8 assay in cell line RBE treated with cordycepin was shown. The mean \pm SD of triplicate experiments were plotted, ${ }^{* * *} P<0.001$. 


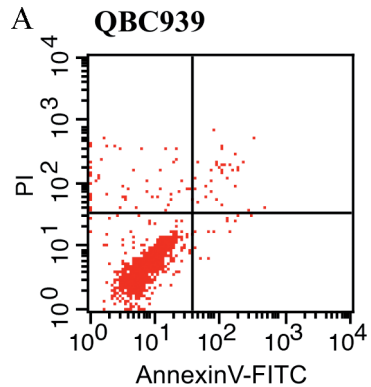

$\mathrm{NC}$

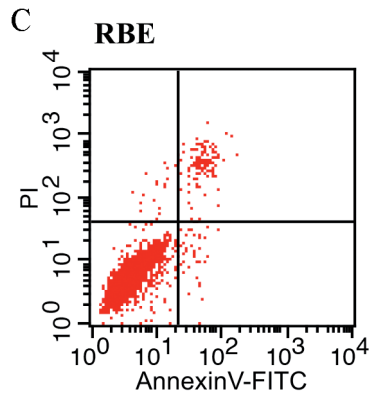

$\mathrm{NC}$

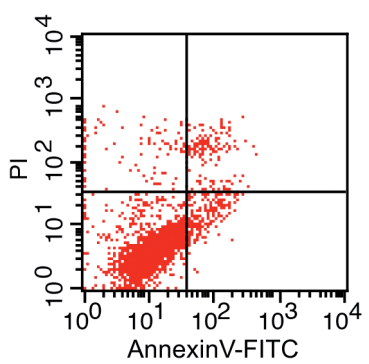

Cordycepin

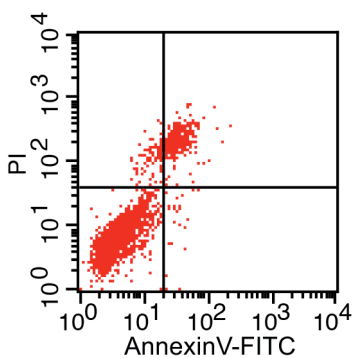

Cordycepin

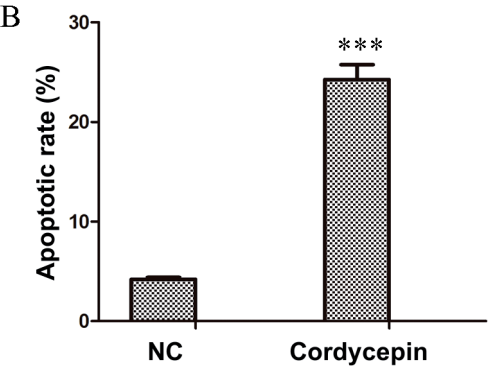

D

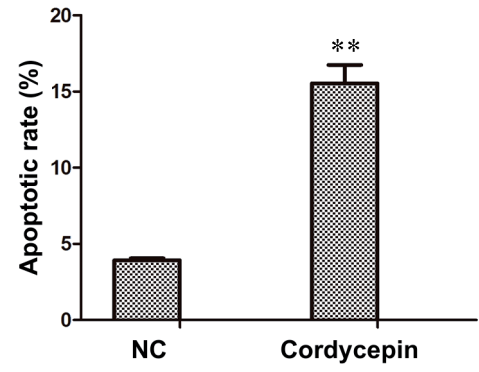

Figure 2. Cordycepin induces apoptosis in human CCA cell. (A and B) Flow cytometric analysis for apoptosis in cell line QBC939 treated with cordycepin was shown. (C and D) Flow cytometric analysis for apoptosis in cell line RBE treated with cordycepin was shown. The mean \pm SD of triplicate experiments were plotted, ${ }^{\star *} P<0.01,{ }^{\star * \star} P<0.001$.
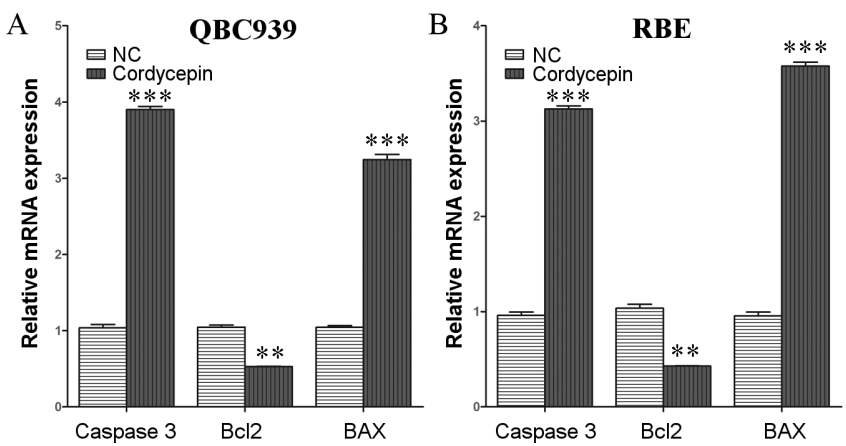

C

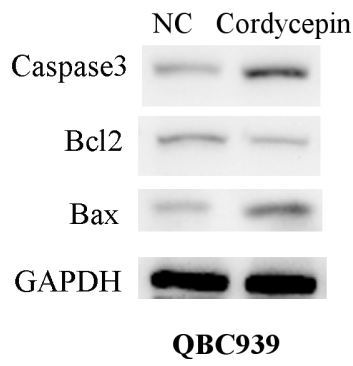

D

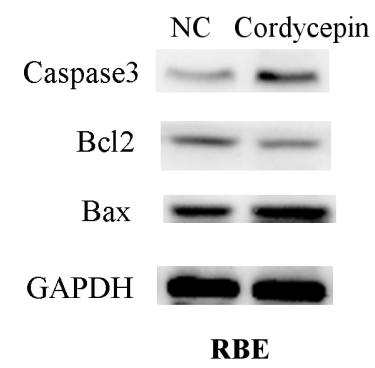

Figure 3. Cordycepin regulates the expression levels of Caspase-3, Bcl-2 and Bax. (A and B) The mRNA levels of Caspase-3, Bcl-2 and Bax in human CCA cell line QBC939 and RBE treated with cordycepin were detected by $\mathrm{qRT}$ PCR. (C and D) The protein levels of Caspase-3, Bcl-2 and Bax in cell line QBC939 and RBE treated with cordycepin were detected by western blot. The mean \pm SD of triplicate experiments were plotted, ${ }^{\star *} P<0.01,{ }^{* *} P<0.001$.

in methanol and stained with $0.1 \%$ crystal violet solution. And we counted the visible colonies which containing at least 50 cells. Each experiment was conducted in triplicate.

Flow cytometric analysis for apoptosis. Human CCA cell was treated with cordycepin for 48 hours. Then the cells were harvested and stained with the AnnexinV/PI double staining kit (BD Biosciences, USA) according to the manufacturer's protocol. Apoptotic cells were measured by flow cytometry on a FACScan (Beckman Instruments, USA). Each experiment was conducted in triplicate.

Western blot. After treatment with cordycepin for human CCA cell for 48 hours, the cells were harvested and lysed in RIPA buffer (Beyotime, China) supplemented with PMSF (Beyotime, China). Subsequently, protein samples were separated by SDS-PAGE gel and transferred to polyvinylidenedifluoride (PVDF, Millipore, USA). Membranes were incubated with anti-Caspase-3(1:1000; Cell Signaling Technology, USA), anti-Bcl2 (1:1000; Cell Signaling Technology, USA), anti-Bax(1:1000; Cell Signaling Technology, USA) and anti-GAPDH antibodies (1:5000; Proteintech, China) at $4^{\circ}$ Covernight, followed by incubation with horseradish peroxidase-conjugated secondary antibody (1:1000; Beyotime, China) and visualized by the Gel Doc 2000 (Bio-Rad, USA).

Tumor xenograft experiment. QBC939cells $(100 \mathrm{ml}$, $1 \times 10^{6}$ cells) were collected and injected subcutaneously into each 4 -week-old male nude mouse (10 micetotally, 5 mice for each group). The first group received an intraperitoneally (i.p.) injection of DMEM high-glucose medium each day. The 
other group was administered an i.p. injection of cordycepin at $40 \mathrm{mg} / \mathrm{kg}$ every day. Mice were monitored daily, and the tumor volumes were assessed $\left(0.5 \times\right.$ length $\times$ width $\left.^{2}\right)$ per 3 days. After 15 days, mice were sacrificed, and all tumor grafts were excised and weighed.

Statistical analysis. All statistical analyses were conducted using SPSS 19.0 software (SPSS, USA). The independent sample $t$-test was used for analyzing the differences between groups. All data were presented as mean \pm standard deviation. A two-sided value of $P<0.05$ was considered statistically significant.

\section{Results}

Cordycepin inhibits proliferation in human CCA cell in vitro. To explore the effect of cordycepin on cell proliferation, we conducted the colony formation assay and the results indicated that the number of colonies of QBC939 and RBE cells treated with cordycepin were both significantly fewer than that of the control (Figure 1A-1D). To further confirm the effect of cordycepin in QBC939 and RBE cells, we conducted CCK- 8 assay and observed the similar phenomenon that the cell proliferation of QBC939 and RBE cells were both significantly inhibited by cordycepin (Figure 1E, 1F). Taken these results together, cordycepin is capable of inhibiting the tumorigenicity of human CCA cell in vitro.

Cordycepin induces apoptosis in human CCA cells. To further explore whether cordycepin mediated inhibition of human CCA cell proliferation was resulted from the induction of apoptosis, we conducted the flow cytometric analysis in QBC939 and RBE cells. Flow cytometric analysis is one of the most effective methods to access the apoptotic cells after drug treatment. As shown, when compared with their control, cordycepin treatment significantly increased the population of apoptotic cells of QBC939 and RBE cells (Figure 2).

However, the underlying mechanism of cordycepin induces human CCA cell apoptosis remains unknown. Next, we conducted the qRT-PCR and western blot assays to access the expression levels of Caspase-3, Bcl-2 and Bax in cordycepin treated QBC939 and RBE cells. As is well known, the activation of Caspase- 3 plays a central role in apoptotic progress [13]. As shown in Figure 3, both mRNA and protein levels of Caspase-3 were increased in cordycepin treated QBC939 and RBE cells. Furthermore, Bcl-2 (anti-apoptosis) and Bax (pro-apoptosis) are members of Bcl-2 family, which plays an essential role in the execution of apoptosis [14]. Our results indicated that the mRNA and protein levels of $\mathrm{Bcl}-2$ were both decreased in cordycepin treated QBC939 and RBE cells, while the opposite phenomenon was observed in Bax (Figure 3). Taken together, these data suggested that cordycepin induces human CCA cell apoptosis by regulating Caspase- 3 and Bcl-2 family members.

Cordycepin suppresses tumor growth in vivo. Yoshikawa et al. intragastrically or intraperitoneally administered mice cordycepin at the maximal tolerant dose, and they
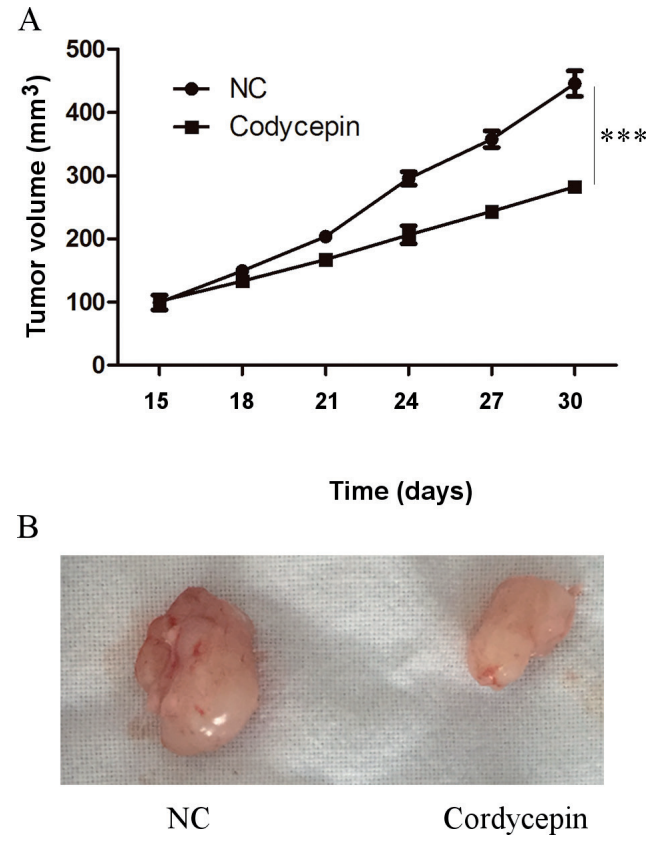

Figure 4. Cordycepin suppresses tumor growth in vivo. (A) Growth curves of tumors in nude mice. Tumor diameters were assessed per three days. (B) The size of the xenograft tumor derived from cordycepin treated QBC939 cells and DMEM high-glucose medium treated QBC939 cells in nude mice were compared. The mean \pm SD of triplicate experiments were plotted, ${ }^{* *} P<0.001$.

did not find the bodyweight loss or systemic toxicity in the mice [15]. Considering that cordycepin could inhibit human CCA cell growth in vitro, in this part, we further explore the effect of cordycepin on the tumor growth in vivo. As shown in Figure 4, the tumor growth in nude mice injected with QBC939 cells was significantly inhibited after treatment with cordycepin compared with the mice injected with DMEM high-glucose medium alone. Thus, these data suggested that cordycepin could inhibit the tumorigenicity of human CCA cell in vivo.

\section{Discussion}

In the present study, we proposed that the growth of human CCA cell could be inhibited by cordycepin in vitro and in vivo. And this inhibition was related to the induction of apoptosis by cordycepin. Furthermore, our results indicated that cordycepin could promote human CCA cell apoptosis via the activation of Caspase- 3 and the modulation of Bcl-2 family in the mRNA and protein level.

One of the most important effects of cordycepin on cancer is the inhibition of cell proliferation. Previous studies suggested that cordycepin inhibits cyclin A2, cyclin E and CDK2 expression that results in the arrestment of cell cycle in S-phase in leukemia cells, suppressing cell proliferation [16]. In addition, cordycepin has been reported to up-regulate p21WAF1 expres- 
sion and induce the phosphorylation of $\mathrm{JNK}$, in regulating the G2/M-phase arrestment and inhibiting cell proliferation in human bladder cancer cells [17]. Not only in human cancers, via the activation of adenosine $\mathrm{A} 3$ receptor, cordycepin could also inhibit mouse lung cancer cells proliferation [18]. These are consistent with our finding that cordycepin inhibits human CCA cell growth by colony formation assay and CCK-8 assay in vitro, and tumor xenograft experiment in vivo. And we supposed that the effect of cordycepin on human CCA cell growth is associated with the cell cycle arrestment or several pathways activation.

Except the arrestment of cell cycle, cell growth inhibition could also be resulted from the induction of apoptosis. However, there have been reported that cordycepin is involved in cell apoptosis in multiple human cancers. Hwang et al. found that cordycepin down-regulated ERK, induced the phosphorylation of JNK, and promoted apoptosis in renal cancer cells [19]. As known to us, the caspase pathway plays an important role in the activation of cell apoptosis. Tian et al. reported that cordycepin promote cell apoptosis by binding the DR3 receptor and activating Caspase-8/3 [20]. Caspase-3 is an executioner caspase and has no activity until it is cleaved by an initiator caspase after the apoptotic signaling has been activated [21]. In this study, the results indicated that cordycepin treatment promoted the human CCA cell apoptosis, and Caspase- 3 was increased in cordycepin treated cells. And we believe that Caspase-3 participates in the cordycepin induced apoptosis in CCA. Moreover, the Bcl-2 family is the key mediator of the intrinsic (mitochondrial) pathway, which includes some of the most extensive-studied proteins, such as Bcl-2, the antiapoptotic protein, Bax, the pro-apoptotic protein [22]. The ratio of $\mathrm{Bcl}-2 / \mathrm{Bax}$ is usually used to assess the occurrence of apoptosis. Our data showed that the expression of $\mathrm{Bcl}-2$ was decreased in cordycepin treated human CCA cell, while conversely in Bax. And the alteration of $\mathrm{Bcl}-2$ and $\mathrm{Bax}$ is according to that of Caspase- 3 .

In summary, this study confirmed that cordycepin is functional of inhibiting the human CCA cell growth and apoptosis. The CCK-8, colony formation andtumor xenograft assays entirely indicated that cordycepin has the ability to inhibit cell proliferation in vitro and in vivo. Flow cytometric analysis indicated that cordycepin promotes apoptosis. Moreover, qRT-PCR and western blot assays indicated that cordycepin induces apoptosis by mediating Caspase- 3 and Bcl-2 family members. We believed that the present study provided some novel information, and further studies about the underlying mechanism were needed.

Acknowledgments: We thank to all the laboratory members for continuous technical advice and helpful discussion.

\section{References}

[1] HRAD V, ABEBE Y, ALI SH, VELGERSDYK J, AL HALLAK $\mathrm{M}$ et al. Risk and Surveillance of Cancers in Primary Biliary
Tract Disease. Gastroenterol Res Pract 2016; 2016: 3432640. https://doi.org/10.1155/2016/3432640

[2] RAZUMILAVA N, GORES GJ. Cholangiocarcinoma. Lancet 2014; 383: 2168-2179. https://doi.org/10.1016/S01406736(13)61903-0

[3] YANG Y, LIU Y, HE JC, WANG JM, SCHEMMER P et al. 14-3-3zeta and aPKC-iota synergistically facilitate epithelial-mesenchymal transition of cholangiocarcinoma via GSK-3beta/snail signaling pathway. Oncotarget 2016; 7: 55191-55210. https://doi.org/10.18632/oncotarget.10483

[4] WANG Y, CUI W, FAN W, ZHANG Y, YAO W et al. Percutaneous intraductal radiofrequency ablation in the management of unresectable Bismuth types III and IV hilar cholangiocarcinoma. Oncotarget 2016; 7: 53911-53920. https://doi.org/10.18632/oncotarget.10116

[5] REN Z, CUI J, HUO Z, XUE J, CUI H et al. Cordycepin suppresses TNF-alpha-induced NF-kappaB activation by reducing p65 transcriptional activity, inhibiting IkappaBalpha phosphorylation, and blocking IKKgamma ubiquitination. Int Immunopharmacol 2012; 14: 698-703. https://doi. org/10.1016/j.intimp.2012.10.008

[6] YAO WL, KO BS, LIU TA, LIANG SM, LIU CC et al. Cordycepin suppresses integrin/FAK signaling and epithelial-mesenchymal transition in hepatocellular carcinoma. Anticancer Agents Med Chem 2014; 14:29-34. https://doi. org/10.2174/18715206113139990305

[7] TAO X, NING Y, ZHAO X, PAN T. The effects of cordycepin on the cell proliferation, migration and apoptosis in human lung cancer cell lines A549 and NCI-H460.J Pharm Pharmacol 2016;68: 901-911. https://doi.org/10.1111/jphp.12544

[8] WANG Z, WU X, LIANG YN, WANG L, SONG ZX et al.Cordycepin Induces Apoptosis and Inhibits Proliferation of Human Lung Cancer Cell Line H1975 via Inhibiting the Phosphorylation of EGFR. Molecules 2016; 21: pii: E1267. https://doi.org/10.3390/molecules21101267

[9] LEE HJ, BURGER P, VOGEL M, FRIESE K, BRUNING A. The nucleoside antagonist cordycepin causes DNA double strand breaks in breast cancer cells. Invest New Drugs 2012;30: 1917-1925. https://doi.org/10.1007/s10637-012-9859-x

[10] CHOI S, LIM MH, KIM KM, JEON BH, SONG WO et al. Cordycepin-induced apoptosis and autophagy in breast cancer cells are independent of the estrogen receptor. Toxicol Appl Pharmacol 2011; 257: 165-173. https://doi.org/10.1016/j. taap.2011.08.030

[11] LEE SY, DEBNATH T, KIM SK, LIM BO. Anti-cancer effect and apoptosis induction of cordycepin through DR3 pathway in the human colonic cancer cell HT-29. Food Chem Toxicol 2013; 60: 439-447. https://doi.org/10.1016/j. $\underline{\text { fct.2013.07.068 }}$

[12] SHAO LW, HUANG LH, YAN S, JIN JD, REN SY. Cordycepin induces apoptosis in human liver cancer HepG2 cells through extrinsic and intrinsic signaling pathways. Oncol Lett 2016; 12: 995-1000. https://doi.org/10.3892/ol.2016.4706

[13] WANG J, LI W. Discovery of novel second mitochondriaderived activator of caspase mimetics as selective inhibitor of apoptosis protein inhibitors. J Pharmacol Exp Ther 2014; 349: 319-329. https://doi.org/10.1124/jpet.113.212019 
[14] JUNG EB, LEE CS. Baicalein attenuates proteasome inhibition-induced apoptosis by suppressing the activation of the mitochondrial pathway and the caspase-8- and Bid-dependent pathways. Eur J Pharmacol 2014; 730: 116-124. https://doi. org/10.1016/j.ejphar.2014.02.039

[15] Yoshikawa N, Nakamura K, Yamaguchi Y, Kagota S, Shinozuka $\mathrm{K}$ et al.Antitumour activity of cordycepin in mice. Clin Exp Pharmacol Physiol2004; 31 Suppl 2: S51-53. https://doi. org/10.1111/j.1440-1681.2004.04108.x

[16] LIAO Y, LING J, ZHANG G, LIU F, TAO S et al. Cordycepin induces cell cycle arrest and apoptosis by inducing DNA damage and up-regulation of p53 in Leukemia cells. Cell Cycle 2015; 14: 761-771. https://doi.org/10.1080/15384101 .2014 .1000097

[17] LEE SJ, KIM SK, CHOI WS, KIM WJ, MOON SK. Cordycepin causes $\mathrm{p} 21 \mathrm{WAF} 1$-mediated G2/M cell-cycle arrest by regulating c-Jun $\mathrm{N}$-terminal kinase activation in human bladder cancer cells. Arch Biochem Biophys 2009; 490: 103-109. https://doi.org/10.1016/j.abb.2009.09.001
[18] Nakamura K, Yoshikawa N, Yamaguchi Y, Kagota S, Shinozuka $\mathrm{K}$ et al. Antitumor effect of cordycepin (3'-deoxyadenosine) on mouse melanoma and lung carcinoma cells involves adenosine A3 receptor stimulation. Anticancer Res 2006; 26: 43-47.

[19] HWANG JH, JOO JC, KIM DJ, JO E, YOO HS et al. Cordycepin promotes apoptosis by modulating the ERKJNK signaling pathway via DUSP5 in renal cancer cells. Am J Cancer Res 2016; 6: 1758-1771.

[20] TIAN X, LI Y, SHEN Y, LI Q, WANG Q et al. Apoptosis and inhibition of proliferation of cancer cells induced by cordycepin. Oncol Lett 2015; 10: 595-599. https://doi.org/10.3892/ ol.2015.3273

[21] WALTERS J, POP C, SCOTT FL, DRAG M, SWARTZ P et al. A constitutively active and uninhibitable caspase- 3 zymogen efficiently induces apoptosis. Biochem J 2009; 424: 335-345. https://doi.org/10.1042/BJ20090825

[22] DAI H, MENG XW, KAUFMANN SH. Mitochondrial apoptosis and BH3 mimetics. F1000Res 2016; 5: 2804. https://doi. org/10.12688/f1000research.9629.1 